Arqueología y Sociedad,

№ 22, 2010

\title{
DECAPITACIÓN Y CABEZAS HUMANAS del Valle de Acarí, Perú
}

\author{
Lidio M. Valdez $z^{i}$ \\ Jocelyn S. Williamsii \\ Katrina J. Bettcheriii \\ Lucie Dausse ${ }^{i v}$
}

\section{Resumen}

Las excavaciones arqueológicas efectuadas en Amato, un sitio establecido a inicios del periodo Intermedio Temprano (circa 50 a.C - 300 d.C) en el valle de Acarí de la costa sur del Perú, permitió recuperar dos cabezas humanas en dos contextos diferentes. El primero proviene de las inmediaciones de decenas de esqueletos humanos decapitados, mientras que el segundo fue expuesto asociado al muro perimétrico que fortifica al sitio. Ambas cabezas recibieron un tratamiento similar a las cabezas trofeo; sin embargo, éstas no tienen el orificio en el hueso frontal, como tampoco presentan el foramen magnum alterado, características propias de las cabezas trofeo. Estos dos hallazgos demuestran que por lo menos en el valle de Acarí, la decapitación humana no fue efectuada exclusivamente para obtener cabezas trofeo. Esto implica además, que la decapitación y la toma de las cabezas fueron efectuadas por diversas razones y para cumplir funciones distintas.

\section{Palabras clave}

Costa Sur peruana, valle de Acarí, decapitación y cabezas humanas.

\begin{abstract}
Archaeological excavations at Amato, a site established at the beginning of the Early Intermediate period (circa $50 \mathrm{BC}-300 \mathrm{AD}$ ) in the Acari Valley of the Peruvian south coast, uncovered two isolated human heads from different contexts. One head was found near an area where dozens intentionally decapitated skeletons were recovered. The second head was located in association with the main wall that encloses the site. Both heads were buried in similar fashion to Early Intermediate period south coast trophy heads; however, these heads from Amato were not culturally modified (e.g. perforated frontal bone and/or artificially enlarged foramen magnum). These two isolated heads demonstrate that not all human decapitation in the Acari Valley was for the purposes of securing trophy heads. Based on these findings, we suggest that the purpose and motivation for human decapitation and head-taking in the past was complex.
\end{abstract}

\section{Keywords}

Peruvian south coast, Acari Valley, decapitation and human heads.

i Department of Anthropology, Economics \& Political Sciences, MacEwan University.

Correo electrónico: valdezcardenasl@macewan.ca

ii Department of Anthropology, Trent University.

Correo electrónico: jocelynwilliams@trentu.ca

iii Acari Valley Archaeological Project.

Correo electrónico: kjbettcher@yahoo.es

iv Universite de Paris I Pantheon Sorbonne.

Correo electrónico: lu-dausse@hotmail.fr 
En la América del Sur, la costa sur del Perú es una de las pocas regiones donde en el pasado -y más que en cualquiera otra región- se practicó la decapitación humana y la subsiguiente toma de cabezas. Si bien dicha práctica se efectuó en otras regiones de los Andes a lo largo de los tiempos, la investigación arqueológica demuestra que fue en la costa sur y de manera particular durante el periodo Intermedio Temprano (circa 50 a.C. - 600 d.C.) que la decapitación y la obtención de las cabezas alcanzó mayor énfasis por razones que siguen siendo materia de estudios científicos (Silverman 1988; Proulx 1989, 2001; Silverman \& Proulx 2002; Verano 1995, 2003). La evidencia arqueológica que atestiguan la decapitación humana se manifiesta mediante la presencia física de las mismas cabezas humanas, identificadas como "trofeos" (Baraybar 1987; Kowta 1987; Drusini \& Baraybar 1991; Forgey \& Williams 2003, 2005; Proulx 1971, 2001; Browne, Silverman \& García 1993; Verano 2001; Neira Avendaño \& Coelho 1972/73; Knudson, et al, 2008), además de cuerpos decapitados (DeLeonardis 2000; Conlee 2007; Valdez 2008, 2009a). La iconografía Nasca también ilustra con cierta recurrencia la representación de las cabezas y por lo menos en una ocasión el mismo acto de la decapitación es escenificado (Verano 1995: Figura 11). Al ser representados en la iconografía Nasca, las cabezas son portadas por figuras antropomorfas, identificados como seres míticos (Proulx 2001).

Los especialistas reconocen que Max Uhle fue el primero en identificar a las referidas cabezas como "trofeos" (Browne, Silverman \& García 1993: 275). Siguiendo dicha identificación, una cabeza trofeo debe presentar como mínimo un orificio en el hueso frontal y tener la sección del foramen magnum expandido, o en su efecto todo el hueso occipital removido (Browne, Silverman \& García 1993: 275; Drusini \& Baraybar 1991: 251; Silverman \& Proulx 2002: 229; Verano 2003: 525; Williams, Forgey \& Klarich 2001: 2). Además, se sostiene que el engrandecimiento del formen magnum fue intencional y hecha para remover las partes blandas del interior del cráneo; mientras tanto, el orificio en el hueso frontal también fue intencional y sirvió para incrustar una cuerda que permita transportar el trofeo.

No obstante que la ocurrencia de cuerpos con signos indiscutibles de decapitación sigue siendo limitado (DeLeonardis 2000; Conle 2007), existe la tendencia a asumir que el acto de la decapitación fue efectuada para obtener cabezas humanas que fueron transformadas en trofeos. Importante es recordar, por lo tanto, que de acuerdo a Carmichael (1988: 291), no toda cabeza trofeo resultó de la decapitación. Ocasionalmente las cabezas de individuos ya muertos también habrían sido recuperados y transformados en trofeos. Esta observación revela que la práctica de obtener cabezas humanas no fue tan simple y denota definitivamente que no toda cabeza trofeo representa una decapitación. Al mismo tiempo, así como recientes estudios arqueológicos realizados en el sitio de Amato del valle de Acarí vienen demostrando (Valdez 2006), la obtención de las cabezas no fue efectuada única y exclusivamente para los propósitos de transformarlos en cabezas trofeo. Por lo tanto, el objetivo de este trabajo es presentar la nueva evidencia procedente de Amato y discutir sus implicancias.

\section{El Sitio Arqueológico de Amato}

Amato es un sitio ubicado en la parte superior media del valle de Acarí, formado por el río del mismo nombre y ubicado inmediatamente al sur de la cuenca del Río Grande (Fig. 1). El sitio fue primero reportado por Rowe (1963), quien lo consideró como un asentamiento perteneciente al periodo Intermedio Temprano (Valdez 1998). Una característica sobresaliente de Amato - y de los otros sitios contemporáneos a éste - es la presencia de grandes muros perimétricos (Fig. 2) que encierran por completo al sitio arqueológico (Valdez 2006: 5, 2009a: 393).

En 1987 el sitio fue visitado por el primer autor en compañía de Francis A. Riddell, llegándose a observar un recinto cuadrado ubicado en la parte central del sitio (Riddell y Valdez 


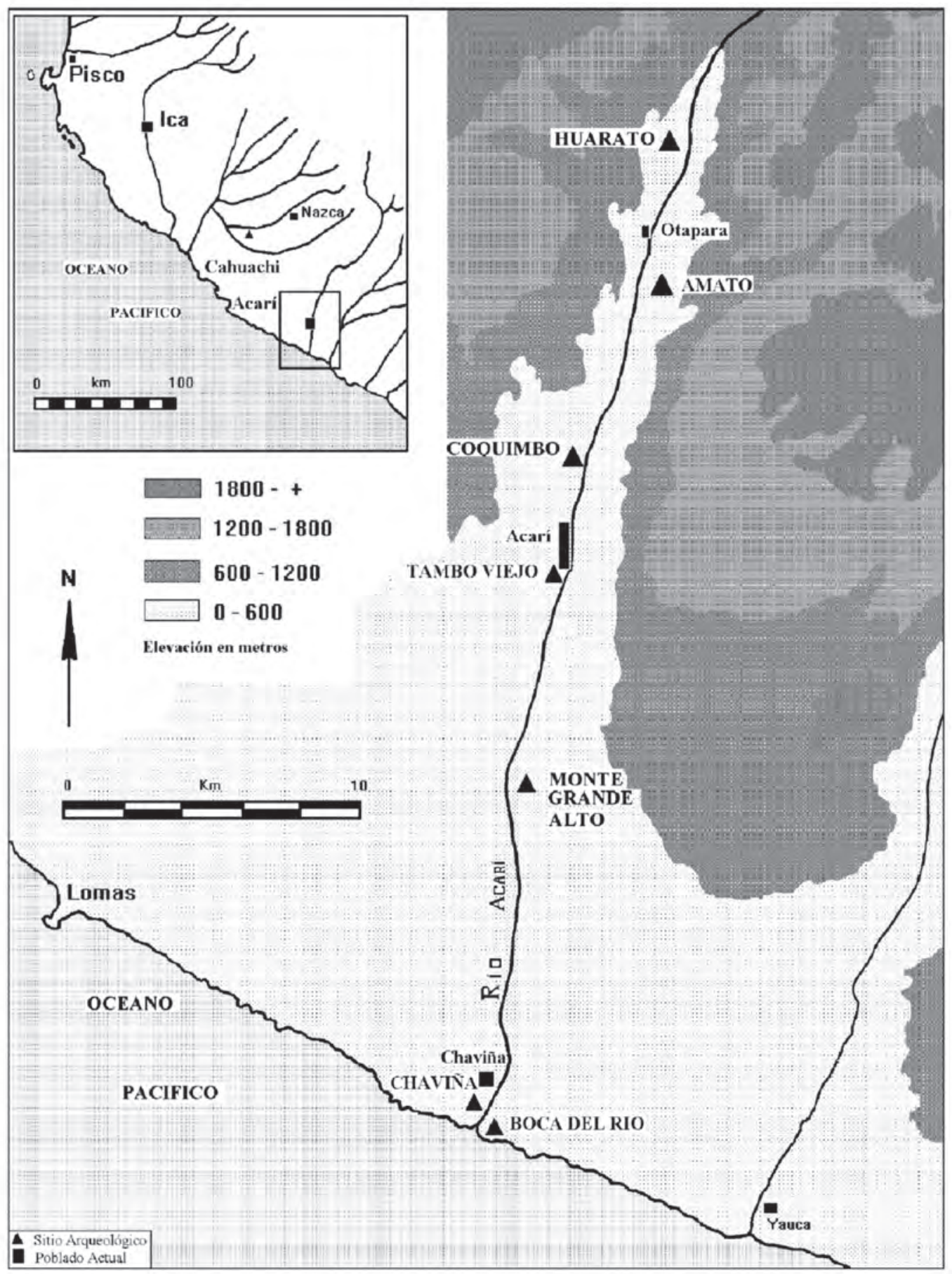

Figura 1. Ubicación de Amato en el valle de Acarí de la costa sur peruana.

(Dibujo por L. Valdez). 




Figura 2. Sitio arqueológico de Amato con indicación del recinto rectangular; las flechas indican la ubicación de los hallazgos (Dibujo por L. Valdez)

1998). Dicho recinto encierra otra estructura más pequeña y de forma rectangular. Durante la inspección superficial de la estructura rectangular se llegó a ubicar en el lado interior del muro del lado este, fragmentos de antara y una pieza trabajada de Spondylus. La presencia de estos artefactos, de manera particular del Spondylus, sugirió la particularidad del recinto rectangular, considerándose que tales artefactos ocurren sólo en contexto especiales.

Teniendo en cuenta los antecedentes aquí brevemente referidos, en 2005 se dio inicio con las primeras excavaciones en Amato, estableciéndose varias unidades de $4 \times 4$ metros de dimensión precisamente al interior de la estructura rectangular. $\mathrm{Al} \mathrm{mismo} \mathrm{tiempo,} \mathrm{tres} \mathrm{uni-}$ dades fueron establecidas al lado exterior este de la mencionada estructura. Otras unidades de prueba fueron también establecidas en las afueras (lado este) del recinto cuadrado; una de estas es una establecida en las inmediaciones de la esquina nor-este del muro perimétrico. Este último fue establecido para exponer una sección del referido muro y evaluar aspectos relacionados a su construcción.

El resultado de la primera temporada de trabajo fue diverso y definitivamente único, en tanto que por primera vez en la historia de la arqueología de la costa sur peruana se llegó a exponer decenas de cuerpos humanos con indiscutibles signos de haber sido decapitados y que fueron expuestos al interior de la estructura rectangular (Valdez 2008, 2009b, 2009c). En general, el estudio osteológico que acaba de concluirse indica que un total de 71 individuos de ambos sexos y todas las edades fueron decapitadas, aunque una mayoría de las víctimas están en la categoría de adulto-joven (20 - 34 años). Teniendo en cuenta que hallazgos similares nunca han sido reportados en toda la costa sur, la evidencia proveniente de Amato es de particular significado.

Además de los cuerpos decapitados, las excavaciones efectuadas en Amato resultaron en el hallazgo de dos cabezas humanas que son definitivamente distintas de las cabezas trofeo. Considerando que se desconoce de la existencia de hallazgos similares, en el resto de este trabajo describimos el contexto de las cabezas, para luego discutir sus inmediatas implicancias arqueológicas.

\section{Las cabezas humanas de Amato}

\section{Cabeza no. 1}

El primer hallazgo, un cráneo sin la mandíbula, fue ubicado al lado exterior del muro del lado este del recinto rectangular (unidad N14W25) (Figs. 2 y 3). El cráneo se halló a sólo $20 \mathrm{~cm}$ de profundidad. Alrededor del cráneo había un total de seis piedras sentadas en barro, al parecer colocadas para protegerlo. Inmediatamente al lado sur del cráneo también había una deposición de varias raíces de una planta identificada como achira (Canna edulis), colocadas a modo de ofrenda. Importante es anotar que el cráneo mantenía una orientación hacia el lado sur-este y había sido directamente cubierto con arena 
eólica. El cráneo fue hallado en excelente estado de conservación y no presenta los atributos de una cabeza trofeo (Figs. 4 y 5). De acuerdo a la evaluación dental, éste pertenece a un subadulto y cuya edad oscila entre los 8 y 10 años. Este presenta una deformación fronto-occipital, y es notable la abertura de las suturas.

Aproximadamente a un metro al este del cráneo se expuso una concentración de varias piedras de tamaños diferentes, todas traídas desde el río, que estaban unidas con argamasa y colocadas formando una especie de pavimento o una cubierta (Figs. 3A y 6) de alguna deposición significante. Para exponer y definir la posible asociación de las piedras, estas fueron retiradas, exponiéndose solamente una densa acumula- ción de arena eólica sin material cultural alguno. Sin embargo, apenas a $60 \mathrm{~cm}$ al lado este y a una profundidad de $55 \mathrm{~cm}$ se llegó a ubicar una olla de cuerpo globular, cuello angosto y labios divergentes (Tumba 1). Además, la olla, dotada de dos asas en posición vertical, había sido colocada en posición vertical, pero su abertura estaba del todo sellada con una laja y argamasa (Fig. 7). Una vez retirado la olla se determinó que este contenía los restos de un infante que primero había sido envuelto en algodón y tejido. La evaluación dental y del crecimiento de los huesos sugiere una edad aproximada de 9 meses ( \pm 6 meses).

Lo notable de este infante es la presencia de una pequeña banda de tejido que estaba coloca-


Figura 3. Ubicación del cráneo expuesto al lado Este del recinto rectangular (Dibujo por L. Valdez) 

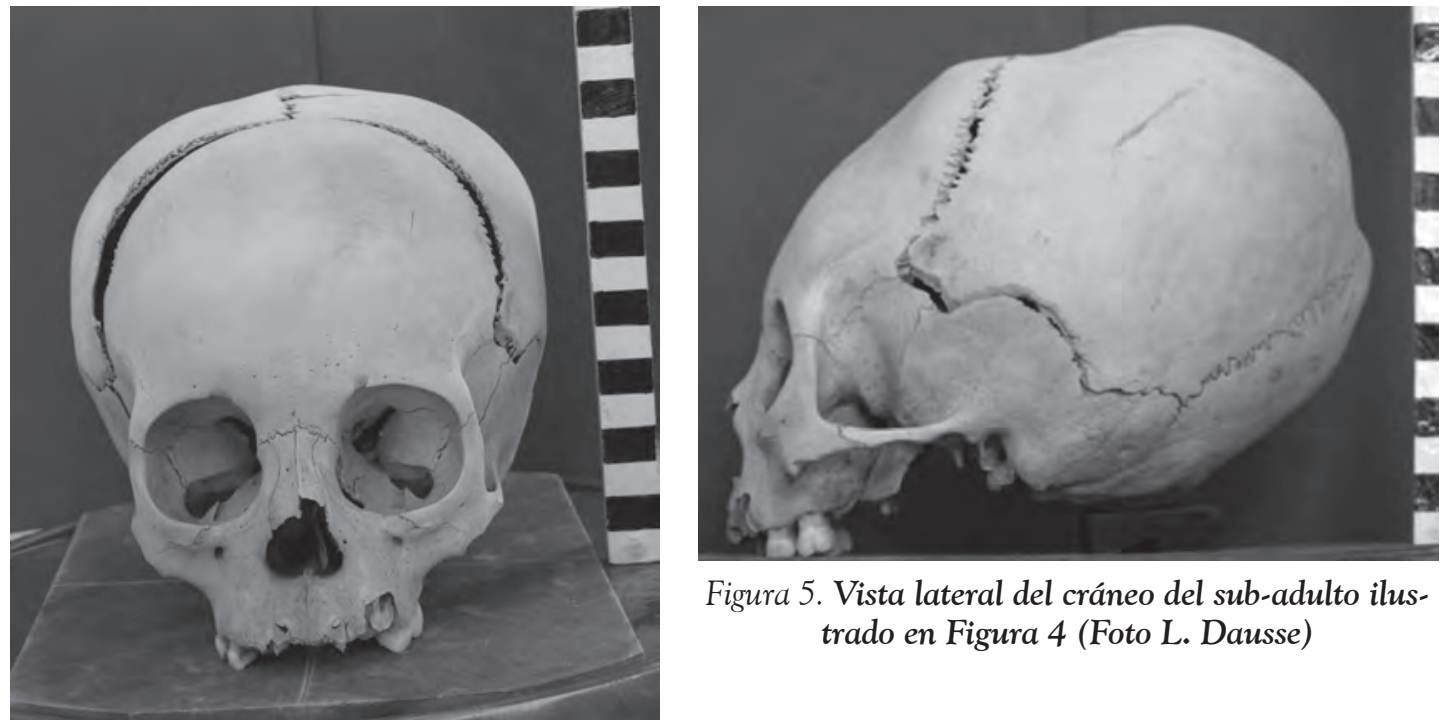

Figura 5. Vista lateral del cráneo del sub-adulto ilustrado en Figura 4 (Foto L. Dausse)

Figura 4. Vista frontal del cráneo de un subadulto ubicado en las afueras del recinto rectangular (Foto L. Valdez)

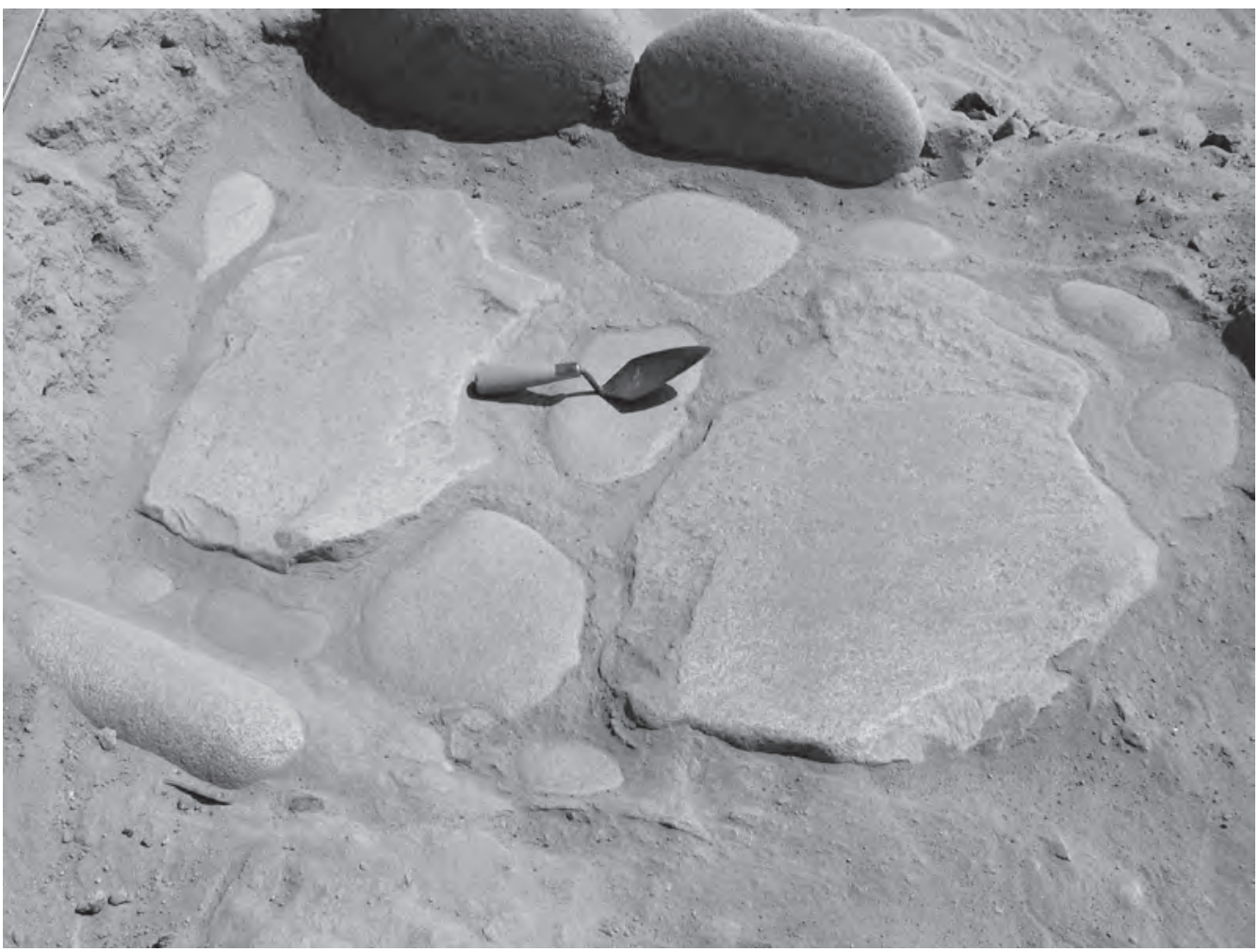

Figura 6. Lajas unidas con argamasa formando una forma de pavimento o cobertura (Foto L. Valdez). 


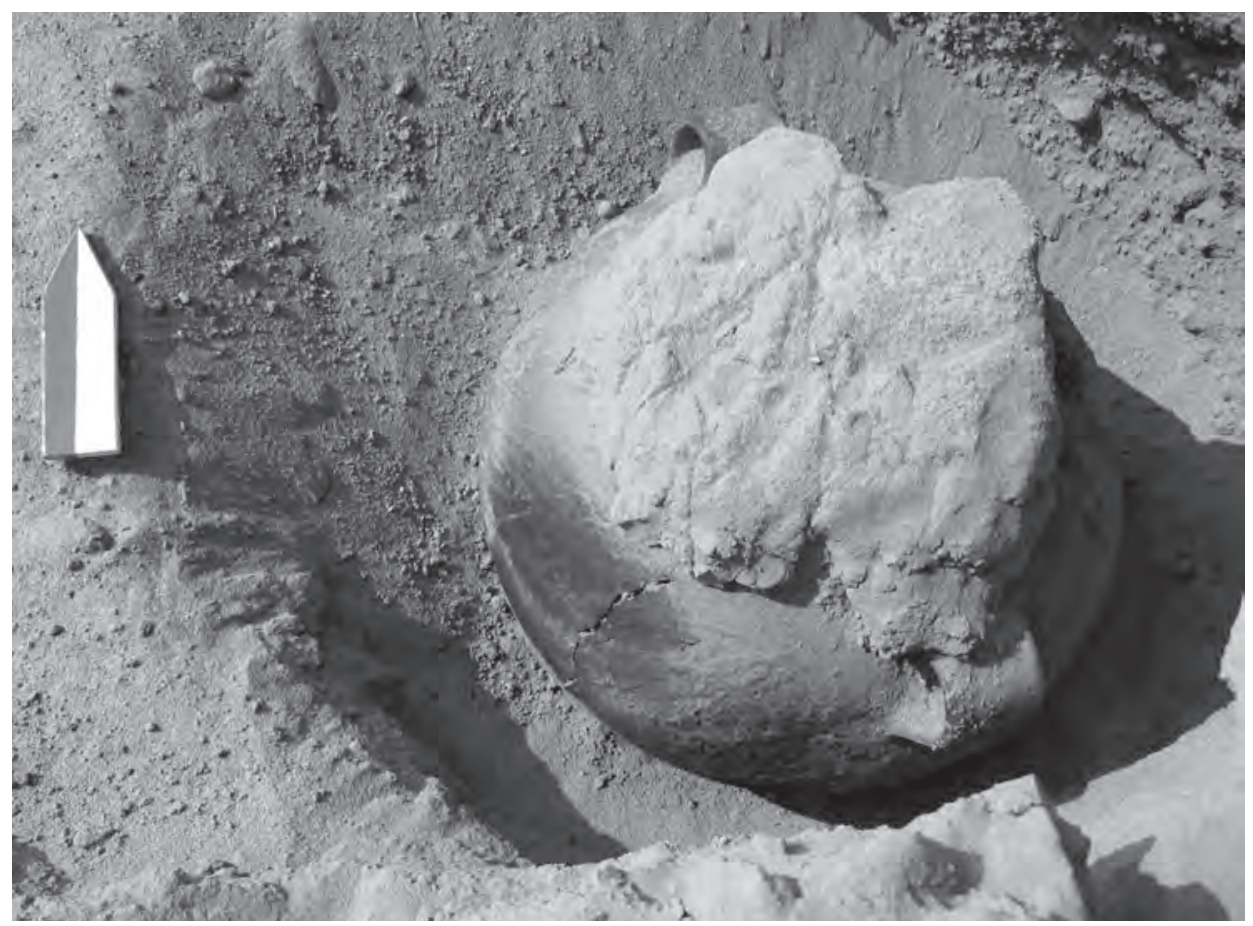

Figura 7. Olla (Tumba 1) descubierta en las inmediaciones de las lajas ilustradas en Figura 6 (Foto L Valdez).

da en la cabeza. La banda está decorada con una serie de pequeñas cabezas (Figs. 8 y 9), que tal vez simbolizan la particular importancia de las cabezas humanas. Además de la banda decorada, una cuenta de Spondylus y un fruto completo de ají (Capsicum sp.) habían sido depositados como ofrendas. Por cuanto otros infantes hasta hoy excavados de los sitios de este periodo en este valle no llevan una banda similar (Valdez 2006) y que además objetos como el Spondylus sólo ocurren en asociación a personajes de importancia, como aquel excavado al interior del recinto rectangular y que estaba asociado a los cuerpos decapitados (Valdez 2009b: 274), parece que la banda decorada y la presencia del Spondylus son indicadores de status. En efecto, todo parece indicar que dentro de las poblaciones del periodo Intermedio Temprano del valle de Acarí, el status social fue heredado.

A un metro al oeste de este hallazgo y a la misma profundidad que la olla previamente mencionada, se llegó a ubicar otra olla (Tumba 2) con las mismas características que el anterior (Figuras $3 \mathrm{~B}$ y 10 ). La vasija estaba rajada y su abertura también sellada con una laja y argamasa. $\mathrm{Al}$ interior de la olla estaban los restos de otro infante, también envuelto en algodón y tejido. Junto al pequeño envoltorio que contenía los restos del infante se hallaron productos de maní (Arachis hypogaea) depositadas como ofrendas. A diferencia del infante anterior, el segundo infante no poseía una banda similar (Fig. 11), hecho que deja abierta la posibilidad que la banda decorada tal vez es símbolo de status social. La evaluación dental y del crecimiento de los huesos sugiere una edad aproximada de 18 meses ( \pm 6 meses). Importante es anotar, y a diferencia del infante anterior, este segundo infante presenta patologías. Por ejemplo, los huesos radio y cúbito, peroné y tibia están deformados (relativamente anchos), y este también es el caso de los huesos metatarsianos (Fig. 12). Los dientes incisivos también presentan defectos al parecer como resultado de la carencia de minerales. 



Figura 9. Detalle de la banda decorada asociada al infante cuya cabeza se ilustra en la Figura 8 (Foto L. Valdez).

Figura 8. Cabeza de un infante de Tumba 1 ilustrada en Figura 7; observe que la cabeza lleva una banda decorada (Foto L. Valdez).



Figura 10. Olla (Tumba 2) ubicada al lado oeste de la olla ilustrada en la Figura 7 (Foto L. Valdez). 


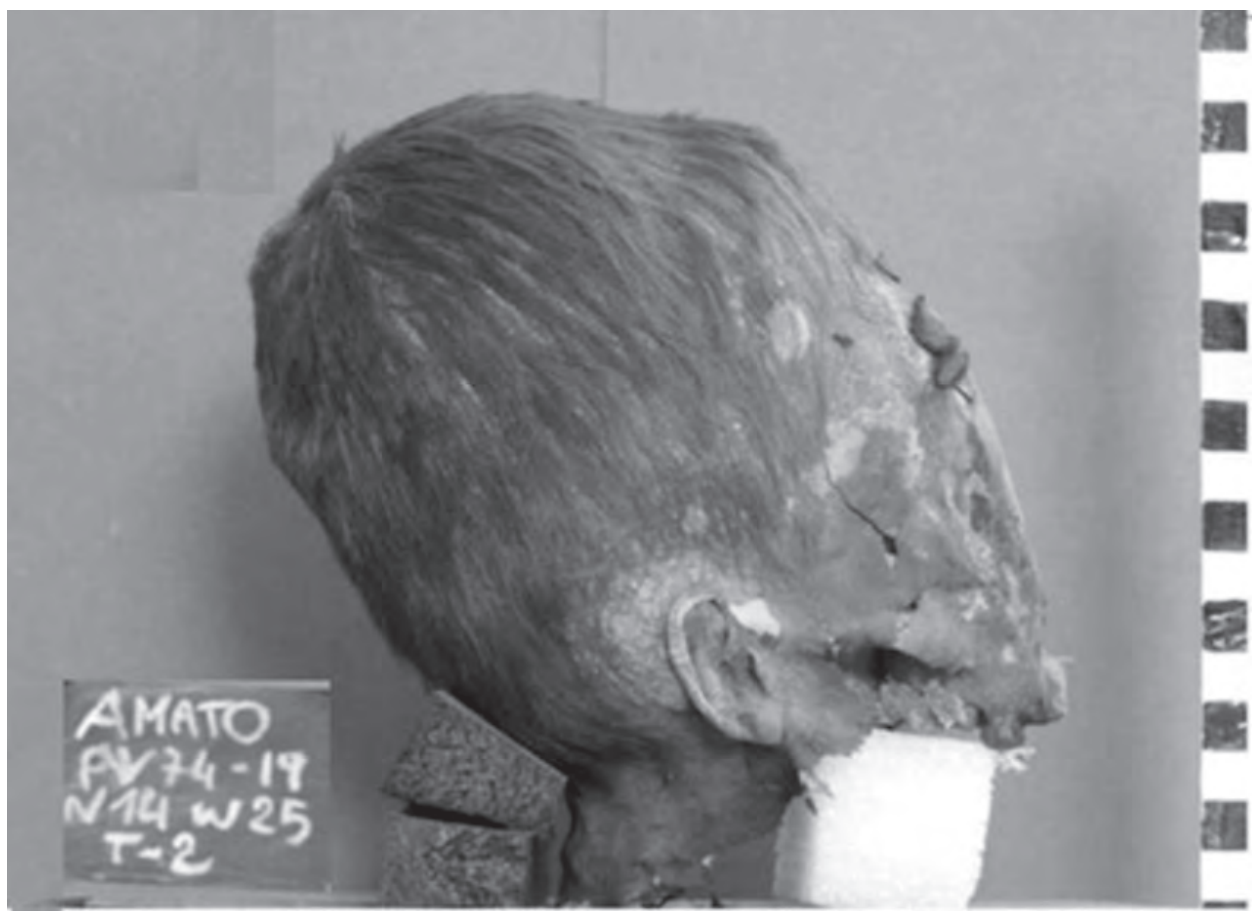

Figura 11. Cabeza del infante de Tumba 2 (Foto L Dausse).

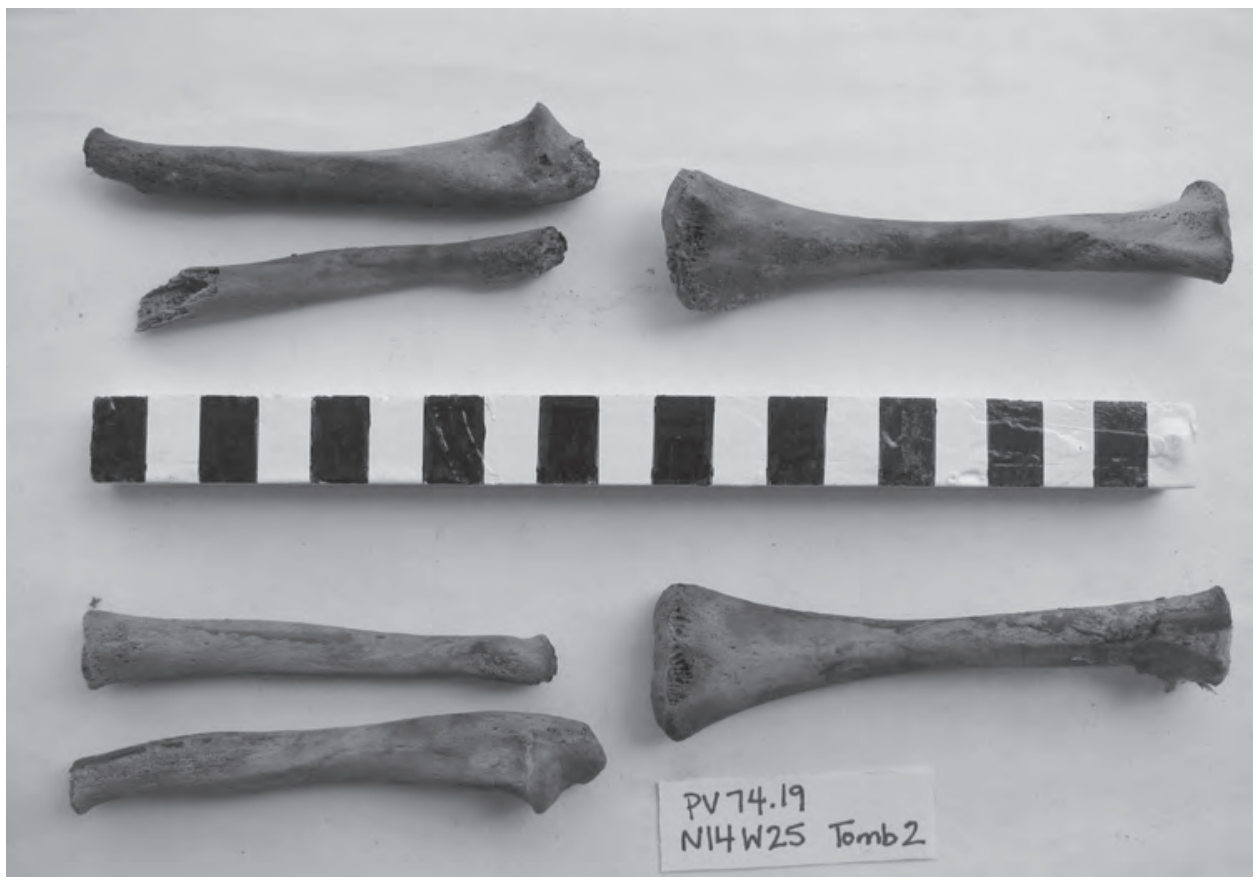

Figura 12. Huesos largos deformados del infante de la segunda olla ilustrada en la figura 10 (Foto L. Valdez). 


\section{Cabeza no. 2}

El segundo hallazgo se trata de una cabeza que a diferencia del anterior incluye la piel momificada y el cabello. Este fue hallado en el lado interior del muro perimétrico, en las inmediaciones de la esquina nor-este del sitio (Fig. 2). Esta vez, la cabeza había sido cubierta en algodón y envuelta en un pequeño manto y los extremos del mismo atados con una cuerda larga (Figs. 13 y 14). Luego, la cabeza había sido colocada al interior de una olla similar al que contenía a los restos de los infantes arriba descritos. Finalmente, la olla conteniendo la cabeza había sido depositada en un pequeño hoyo excavado precisamente a la altura de la base del muro. Previamente, la abertura de la olla había sido sellada con una laja y argamasa. Desafortunadamente fue difícil determinar la orientación de la cabeza por estar ésta envuelta en el tejido y el hecho que el contenido del tejido fue abierto en el laboratorio (Fig.15). A diferencia del cráneo anterior que estaba asociado a raíces de achira, ningún otro artefacto fue hallado en asociación a la cabeza. Por su directa asociación al muro perimétrico, parece que la cabeza fue depositada como una ofrenda dedicatoria al muro.

Por alguna razón, el lado izquierdo de la cara (incluida la parte izquierda de la mandíbula) no está presente (Fig.16). Esto tal vez ocurrió al momento de obtener la cabeza, que tal vez fue de manera violenta. La evaluación del desarrollo dental, así como el crecimiento de los huesos, el desarrollo del hueso occipital y de la placa timpánica (Ubelaker 1978; Scheuer y Black 2000) es consistente con una edad promedio no menor de 8 meses de formación fetal y no mayor de dos meses; en otras palabras, un feto / recién nacido.

\section{Cabezas y Cabezas Trofeo}

Los dos hallazgos aquí referidos son distintos de los propiamente considerados cabezas trofeo, en tanto que ninguno presenta un orificio en el hueso frontal, como tampoco tienen el foramen magnum alterado. Tal como ya se anotó líneas adelante, los especialistas consideran tales atri- butos como los más sobresalientes de una cabeza trofeo (Drusini \& Baraybar 1991: 251; Silverman \& Proulx 2002: 229; Verano 1995: 204, 2003: 525; Williams, Forgey \& Klarich 2001: 2). Por lo tanto, siguiendo el criterio empleado por los especialistas, las dos cabezas provenientes de Amato no podrían ser considerados como trofeos.

La definición empleada por los especialistas de la costa sur en determinar cuál es un trofeo tiene muchas limitaciones. Mientras tal definición permite distinguir una cabeza con el orificio en el hueso frontal y el foramen magnum expandido de otras que no presentan tales atributos y a su vez facilita el discurso de los especialistas, la definición pueda que no necesariamente refleje la ideología e intención de quienes participaron en la obtención de las referidas cabezas. Tal como es discutido por Verano (1995), existen muchos ejemplos provenientes de diferentes sitios y de diferentes periodos donde la cabeza humana ocurre. Al mismo tiempo, existen ejemplos donde otras partes del cuerpo humano también ocurren (Verano 1995: 200; Chacon y Dye 2007). En todos estos casos, la dificultad radica en determinar si dichas partes del cuerpo humano fueron también trofeos.

Al margen de esta limitación de orden semántica, lo sobresaliente de ambas cabezas es el tratamiento que recibieron al momento de ser enterrados. Por ejemplo, el cráneo expuesto en las inmediaciones del recinto rectangular recibió similar tratamiento a las cabezas trofeo excavadas en Chaviña - enterradas en hoyos. La única notable diferencia es que el cráneo de Amato no estaba envuelto en tejido, contrario a los de Chaviña (Neira Avendaño \& Coelho 1972-73). Por su lado, la cabeza hallada junto al muro perimétrico, envuelto en algodón y tejido, y finalmente depositado al interior de una olla, había sido tratada de una forma idéntica a las cinco cabezas trofeo excavadas en Tambo Viejo (Kowta 1987: 66). En ambos casos es notable la intencionalidad de enterrar a las cabezas, la misma que resembla bastante a la forma como las cabezas trofeo fueron enterradas. 


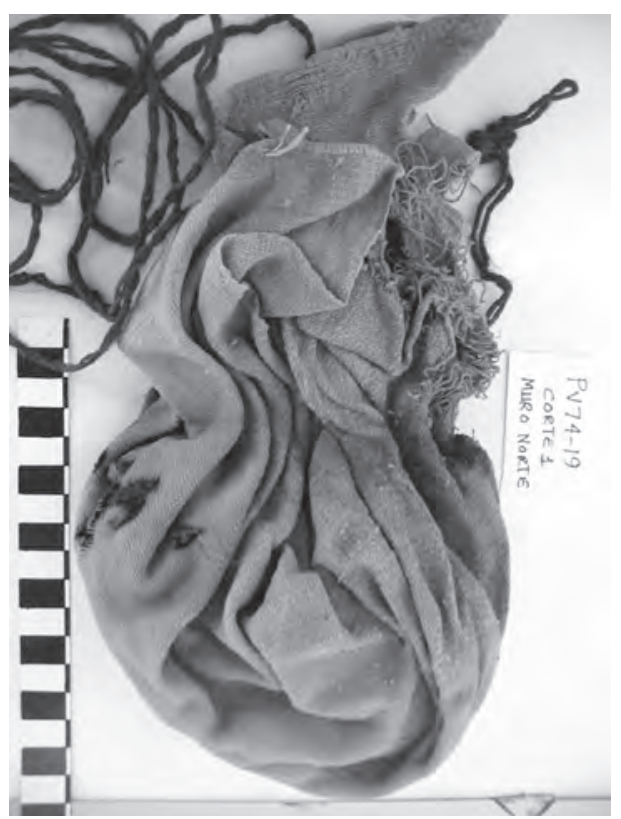

Figura 13. Tejido atado conteniendo la cabeza de un infante hallado en la esquina NorEste de Amato (Foto L. Valdez).

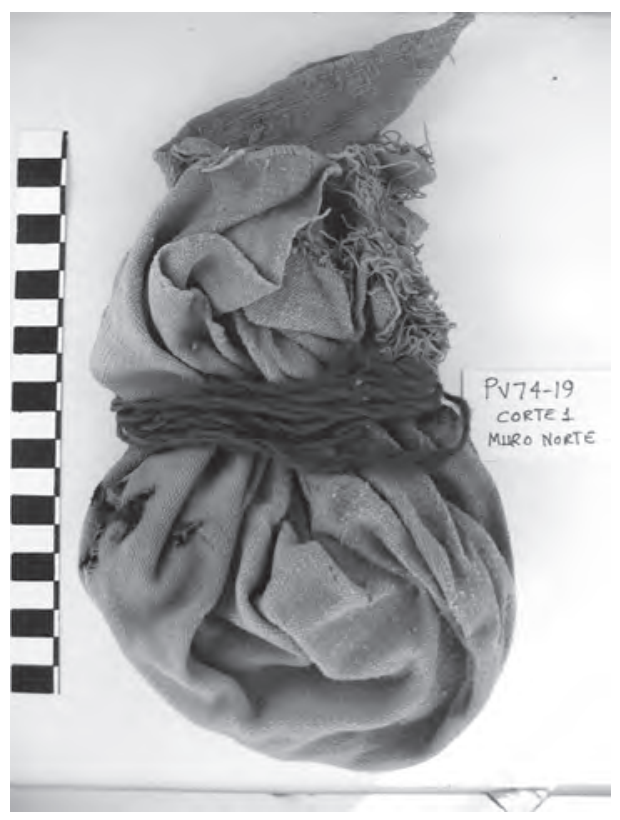

Figura 14. El tejido ilustrado en la Figura 12 una vez retirado la cuerda (Foto L. Valdez).

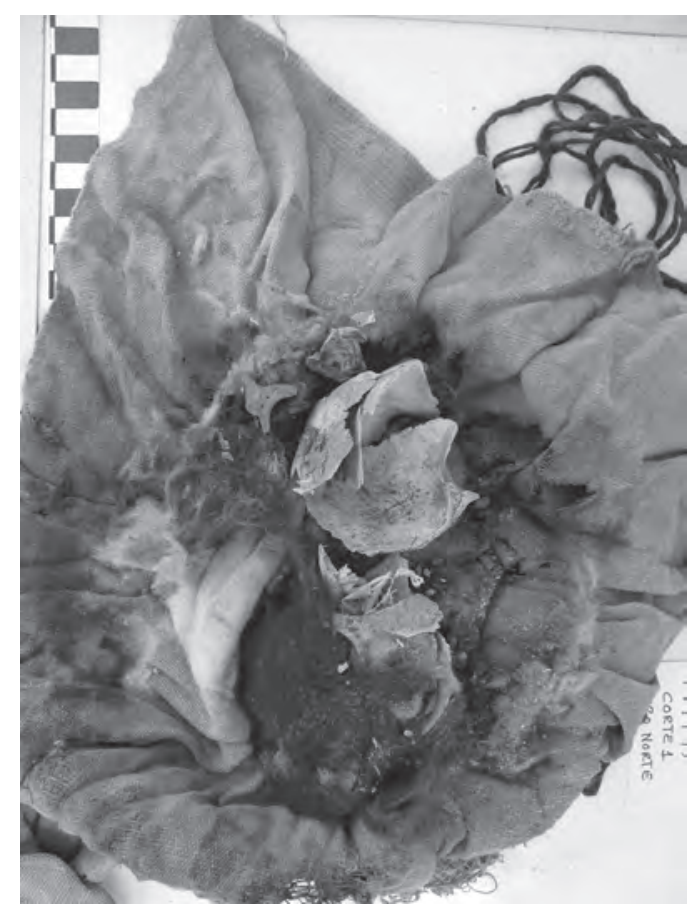

Figura 15. Cabeza fragmentada de un infante hallado al interior del tejido ilustrado en la Figura 13 (Foto L. Valdez). 


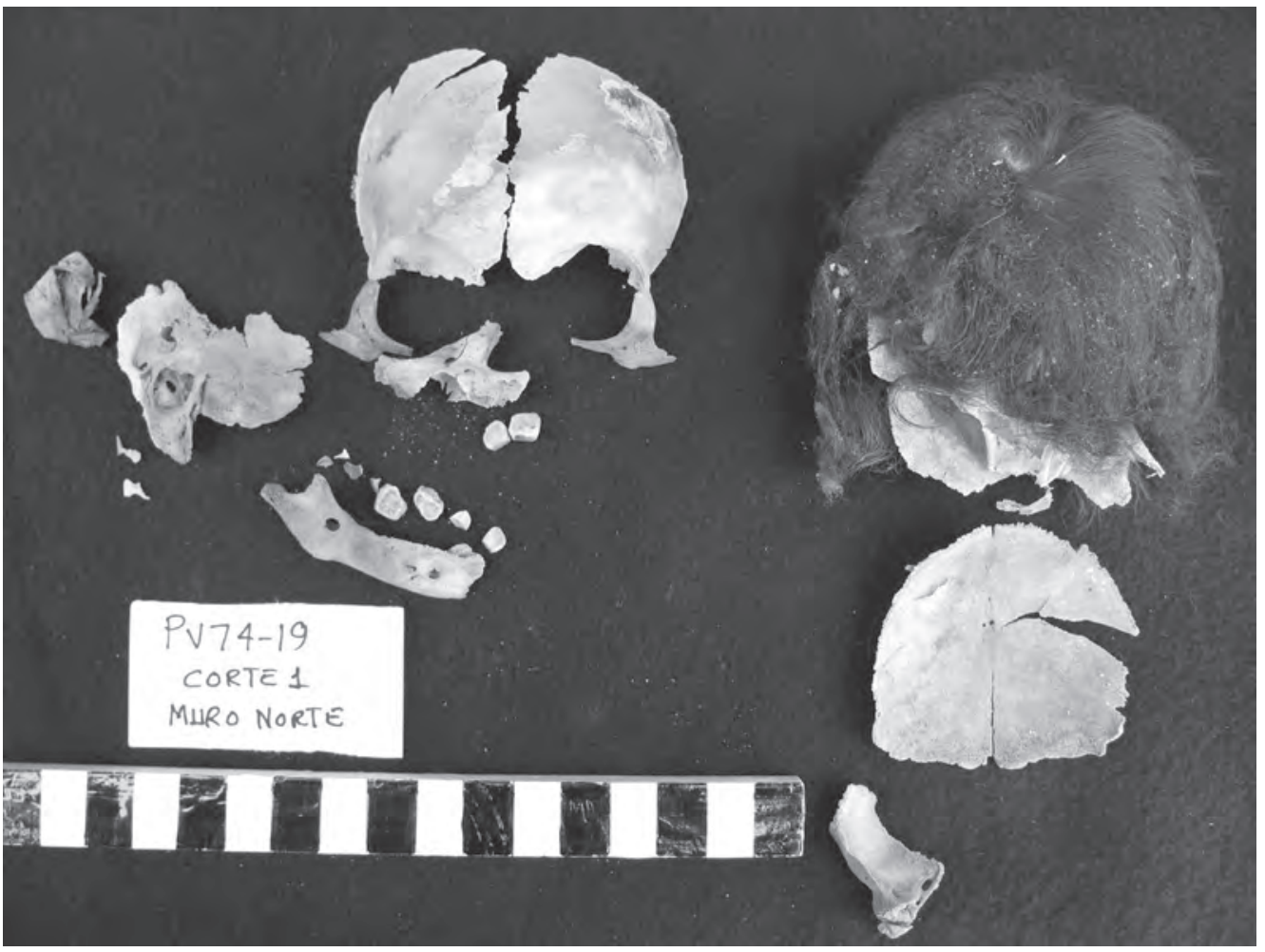

Figura 16. Los huesos fragmentados de la cabeza del infante ilustrado en las figuras 12, 13 y 14 (Foto L. Valdez).

Considerando que hallazgos similares no han sido reportados de ningún sitio del periodo Intermedio Temprano de la costa sur, las dos cabezas provenientes de Amato constituyen las únicas muestras para toda la región. Sin embargo, es oportuno recordar el estado de destrucción de los sitios del periodo Intermedio Temprano a lo largo de toda la costa sur. Del mismo modo, muy pocos sitios Nasca han sido excavados y esta podría ser otra de las razones por las que cabezas similares siguen siendo poco conocidos. También es preciso recordar que un buen número de las mismas cabezas trofeo no han sido recuperadas mediante excavaciones sistemáticas, sino por los huaqueros (Forgey \& Williams 2005: 253). Muchos otros ejemplares de las mismas cabezas trofeo también han sido recuperados de la superficie de los sitios, precisamente porque son fáciles de distinguir. Por el contrario, otros huesos, incluido las cabezas, no siempre son recuperados. Esta podría ser una de las razones por las que cabezas como las de Amato siguen siendo invisibles en el registro arqueológico. Se hace oportuno agregar que en el sitio de Hacha perteneciente al periodo Inicial y ubicado en el valle de Acarí, durante las excavaciones efectuadas en 1986 se llegó a recuperar 3 cabezas humanas pero sin las características de las cabezas trofeo. Dicho hallazgo sugiere que la práctica de obtener cabezas humanas es relativamente antigua en el valle de Acarí.

Así como se mencionó líneas adelante, la iconografía Nasca ilustra con claridad que las supuestas cabezas trofeo carecen de la cuerda incrustada en el hueso frontal. En su lugar, las cabezas son transportadas del cabello. Por ejemplo, los seres míticos representados por Proulx (2001) portan a las así identificadas como cabezas trofeo de los cabellos y no de una cuerda. Esta evidencia sugiere que además de las cabe- 
zas trofeo, otras cabezas como las recientemente expuestas en Amato también existieron, pero que siguen siendo poco visibles en el registro arqueológico posiblemente por las razones arriba anotadas. Del mismo modo, las cabezas representadas en los tejidos Paracas Necrópolis, a menudo identificados como 'cabezas trofeo', no siempre aparecen sujetadas de una cuerda, sino del cabello. De acuerdo a Paul (2000:7 3-76), sólo el 25\% de las cabezas son portados de la cuerda y sugiere que un $75 \%$ de las cabezas representadas no eran trofeos.

Entre otras, la interrogante que surge es por qué algunas cabezas, como las provenientes de Amato, no fueron transformados en trofeos. Definitivamente, edad no fue el criterio determinante en tanto que cabezas trofeos pertenecientes a niños son bien conocidos para la costa sur (ver Neira Avendaño y Coehlo 1972-73; Drusini y Baraybar 1991: 262; Forgey y Williams 2005: 270). Por el momento, y hasta que otros ejemplares similares sean expuestos y se conozca el contexto del que provienen, es posible sostener que la toma de las cabezas humanas en la costa sur se efectuó por diversos razones y para cumplir objetivos diferentes. Así como atestigua la evidencia arqueológica, algunas fueron obviamente transformadas en trofeos, mientras que otras nunca fueron modificadas. Nuevamente, las razones para esta variación siguen siendo inciertas.

Considerando el contexto en el que ambas cabezas fueron halladas, es posible adelantar algunas posibilidades, esperando que estudios posteriores verifiquen esta aseveración. La primera cabeza, hallada en las inmediaciones a dos entierros de infantes, tal vez fue depositado para 'proteger' la tumba de los infantes. La orientación de la cabeza, precisamente en dirección a las tumbas de los infantes, deja esta posibilidad. Por su parte, la cabeza hallada junto al muro perimétrico posiblemente fue una ofrenda dedicatoria, tal vez depositada al completarse la construcción del referido muro. Sin embargo, siempre existe la posibilidad que cabezas similares hayan cumplido varias funciones (Hill 2006:93).

\section{Conclusión}

En este trabajo presentamos una nueva evidencia relacionada a la obtención de cabezas humanas practicada en el valle de Acarí durante las fases iniciales del periodo Intermedio Temprano. No obstante la dificultad en determinar si las cabezas fueron obtenidos mediante la decapitación, la nueva evidencia sugiere que además de las mismas cabezas trofeo, por lo menos en el valle de Acarí se practicó la colección de cabezas humanas. Dichas cabezas nunca fueron transformadas físicamente, contrario a las cabezas trofeo. Sin embargo, por lo menos al momento de ser enterrados, estas si fueron tratadas en la misma forma como las cabezas trofeo. Esto sugiere que tales cabezas también cumplieron funciones importantes. Considerando el contexto de ambos hallazgos, aquí se sugiere que una de las cabezas fue depositada para proteger las tumbas de dos infantes, mientras que la segunda cabeza parece que fue una dedicatoria al muro perimétrico.

El hallazgo de estas dos cabezas también permite sostener que dentro de las poblaciones del periodo Intermedio Temprano Acarí, por lo menos durante sus fases iniciales, las cabezas parecen haber sido obtenidos para cumplir distintas funciones. Tal vez respondiendo a dichas particularidades, algunas tuvieron que ser transformadas en cabezas trofeo, mientras que otras no recibieran el mismo procesamiento. Cuáles fueron las determinantes para decidir qué cabezas debieron ser transformadas y cuáles no, es una interrogante de vital importancia, pero difícil de responder precisamente porque las muestras son limitadas. Esperamos que el hallazgo de restos similares ayude a esclarecer aspectos que por ahora siguen siendo desconocidos.

De la información que se dispone, se puede concluir que la decapitación humana en este valle no fue efectuada exclusivamente para los fines de obtener trofeos. En su lugar, la nueva evidencia sugiere que las cabezas cumplieron muchas funciones y que constituyeron un aspecto de mucho significado dentro de la ideología local. En particular, la asociación de la banda decorada al entierro de un infante sugiere que las cabezas parece que fueron símbolos de poder 
y status. No obstante que el entierro de infantes por lo general, contrario al entierro de adultos, está asociado a ofrendas, este estudio revela por primera vez la asociación de un infante con símbolos que representan cabezas antropomorfas. Esta evidencia parece efectivamente señalar que el status fue heredado.

\section{Agradecimientos}

Los trabajos arqueológicos efectuados en Amato fueron llevados adelante con autorización del Instituto Nacional de Cultura y la colaboración de las autoridades ediles de Acarí. Muchas son las personas que participaron durante los trabajos de campo y enumerarlos sería una lista larga y tal vez incompleta. Sin embargo, quisiéramos expresar nuestro sincero reconocimiento a Rosa Mazuelo, Ángel Iglesias, Alan Chalco, Martin Cantalicio, Eber Meléndez y Román Gutiérrez por las facilidades y ayuda desinteresada que siempre han ofrecido, haciendo de nuestras labores y sobre todo de nuestra estadía en Acarí cómodas y memorables. El primer autor extiende su reconocimiento a la MacEwan University por una beca que facilitó el inicio de los trabajos de laboratorio durante el verano de 2009.

\section{BIBLIOGRAFÍA}

Baraybar, J.P.

1987 "Cabezas trofeo Nasca: nuevas evidencias". Gaceta Arqueológica Andina 15: 6-11.

Browne, D. M., H. Silverman \& R. García

1993 "A cache of 48 Nasca trophy heads from Cerro Carapo, Peru". Latin American Antiquity 4: 274-294.

Carmichael, P. H.

1988 Nasca mortuary customs: Death and ancient society on the south coast of Peru. Doctoral dissertation, Department of Archaeology, University of Calgary, Calgary.

Chacon, R. J. \& D. H. Dye

2007 "Introduction to human trophy taking: an ancient and widespread practice". In, The taking and displaying of human body parts as trophies by Amerindians. Edited by R. J. Chacon and D. H. Dye, pp. 5-31. Srpinger, New York.

Conlee, C.A.

2007 "Decapitation and rebirth: a headless burial from Nasca, Peru". Current Anthropology 48: 438-445.

DeLeonardis, L.

2000 "The body context: interpreting early Nasca decapitated burials". Latin American Antiquity 11 (4): 363-386.

Drusini, A.G. \& J.P. Baraybar

1991 "Anthropological study of Nasca trophy heads". Homo 41:251-265.

Forgey, K. \& S.R. Williams

2003 "Cabezas trofeo Nasca: evidencias osteológicas y arqueológicas de la colección Kroeber". Revista Andina 36: 237-261).

Forgey, K. \& S.R. Williams

2005 "Were Nasca trophy heads war trophies or revered ancestors?" En, Interacting with the Dead: Perspectives on Mortuary Archaeology for the New Millennium, editado por G. F. M. Rakita, J. E. Buikstra, L. A. Beck, \& S. R. Williams, pp. 251-276. University Press of Florida, Gainesville.

Hill, E.

2006 "Moche skulls in cross-cultural perspective". En, Skull Collection, Modification and Decoration, edited by M. Bonogofsky, pp. 91-100. BAR international Series 1539, Oxford.

Knudson, K. J., S. R. Williams, R. Osbornm K. Forgey \& P. R. Williams.

2008 "The geographic origins of Nasca trophy heads using strontium, oxygen, and carbon isotope data". Journal of Anthropological Archaeology 27 (4): 244-257.

Kowta, M.

1987 An Introduction to the Archaeology of the Acari Valley in the south coast region of Peru. California Institute for Peruvian Studies, Sacramento.

Neira Avendaño, M. \& V. P. Coelho

1972-73 "Enterramientos de cabezas de la cultura Nasca”. Revista do Museu Paulista 20: 109-142.

Paul, A.

2000 "Bodiless human heads in Paracas Necrópolis textile iconography". Andean Past 6: 69-94. 
Proulx, D. A.

1971 "Headhunting in Ancient Peru". Archaeology 24(1): 16-21.

1989 "Nasca trophy heads: victims of warfare or ritual sacrifice?" En, Cultures in Conflict: Current Archaeological Perspectives, editado por D.C. Tkaczuk \& B.C. Vivian, pp. 73-85. Archaeological Association of the University of Calgary, Calgary.

2001 "Ritual uses of trophy heads in ancient Nasca society". En, Ritual Sacrifice in Ancient Peru, editado por E. Benson \& A. Cook, pp. 119-136. University of Texas Press, Austin.

2008 "Paracas and Nasca: regional cultures on the south coast of Peru". En, Handbook of South American Archaeology, editado por H. Silverman \& W. H. Isbell, pp. 563-585. Springer, NY.

Riddell, F. A. \& . M. Valdez

1988 Prospecciones Arqueológicas en el valle de Acarí, costa sur del Perú. California Institute for Peruvian Studies, Sacramento.

Rowe, J.H.

1963 "Urban settlements in ancient Peru". Nawpa Pacha 1: 1-27.

Scheuer, L. \& S. Black

2000 Developmental Juvenile Osteology. Academic Press, San Diego.

Silverman, $\mathrm{H}$.

1988 "De la historia Antigua del Perú: la obtención de las cabezas trofeo". Boletín de Lima 58: 49-56.

Silverman, H. \& D.A. Proulx

2002 The Nasca. Blackwell Publishers, Malden, MA.

Ubelaker, D. H.

1978 Human Skeletal Remains: excavation, analysis, interpretation. Manuals in Archaeology No. 2. Tarazacum, Washington.

Valdez, L.M.

2006 "Los vecinos de Nasca: entierros de la tradición Huarato del valle de Aca- rí, Perú". Bulletin de l'Institut Français d'Études Andines 35 (1): 1-20.

2008 "Decapitación Humana en Amato, valle de Acarí, Perú". Boletín de Lima 153, Vol. 30: 68-79.

2009a "Walled settlements, Buffer zones and Human decapitation in the Acari Valley, Peru". Journal of Anthropological Research 65 (3): 389-416.

2009b "La Investigación arqueológica en el valle de Acarí y la contribución de Francis A. Riddell". En, Arqueología del Área Centro Sur Andina: Actas del Simposio Internacional, editado por M.S. Ziólkowski, J. Jennings, L.A. Belan, \& A. Drusini, pp. 255-282. Andes 7, Boletín del Centro de Estudios Precolombinos de la Universidad de Varsovia.

2009c "Conflicto y decapitación humana en Amato, valle de Acarí, Perú". Bulletin de l'Institut Français d'Etudes Andines 38 (2):177-204.

Verano, J.W.

1995 "Where do they rest? The treatment of human offerings and trophies in ancient Peru". En, Tombs for the Living: Andean Mortuary Practices, editado por T. D. Dillehay, pp. 189-227. Dumbarton Oaks, Washington, D.C.

2001 "The physical evidence of human sacrifice in ancient Peru". En, Ritual Sacrifice in Ancient Peru, editado por E. Benson \& A. Cook, pp. 165-184. University of Texas Press, Austin.

2003 "Mummified trophy heads from Peru: diagnostic features and medicolegal significance". Journal of Forensic Sciences 48 (3):525-530.

Williams, S.R., K. Forgey \& E. Klasrich

2001 An Osteological Study of the Nasca Trophy Heads Collected by A. L. Kroeber during the Marshall Field Expeditions to Peru. Fieldiana 33. Field Museum of Natural History, Chicago. 